\title{
Differential associations between neocortical tau pathology and blood flow with cognitive deficits in early-onset vs late-onset Alzheimer's disease
}

\author{
Denise Visser ${ }^{1}$. S Sander C. J. Verfaillie ${ }^{1}$ - Emma E. Wolters ${ }^{1,2} \cdot$ Emma M. Coomans $^{1} \cdot$ Tessa Timmers $^{1,2}$. $^{1}$ \\ Hayel Tuncel ${ }^{1} \cdot$ Ronald Boellaard $^{1}$. Sandeep S. V. Golla ${ }^{1}$ - Albert D. Windhorst ${ }^{1}$. Philip Scheltens ${ }^{2}$. \\ Wiesje M. van der Flier ${ }^{2,3} \cdot$ Bart N. M. van Berckel ${ }^{1} \cdot$ Rik Ossenkoppele $^{2,4}$
}

Received: 17 August 2021 / Accepted: 20 December 2021 / Published online: 8 January 2022

(C) The Author(s) 2022

\begin{abstract}
Purpose Early-onset Alzheimer's disease (EOAD) and late-onset Alzheimer's disease (LOAD) differ in neuropathological burden and type of cognitive deficits. Assessing tau pathology and relative cerebral blood flow (rCBF) measured with $\left[{ }^{18} \mathrm{~F}\right]$ flortaucipir PET in relation to cognition may help explain these differences between EOAD and LOAD.

Methods Seventy-nine amyloid-positive individuals with a clinical diagnosis of AD (EOAD: $n=35$, age-at-PET $=59 \pm 5$, MMSE $=23 \pm 4$; LOAD: $n=44$, age-at-PET $=71 \pm 5$, MMSE $=23 \pm 4$ ) underwent a 130-min dynamic $\left[{ }^{18} \mathrm{~F}\right]$ flortaucipir PET scan and extensive neuropsychological assessment. We extracted binding potentials $\left(\mathrm{BP}_{\mathrm{ND}}\right)$ and $\mathrm{R}_{1}$ (proxy of $\mathrm{rCBF}$ ) from parametric images using receptor parametric mapping, in medial and lateral temporal, parietal, occipital, and frontal regions-of-interest and used nine neuropsychological tests covering memory, attention, language, and executive functioning. We first examined differences between EOAD and LOAD in $\mathrm{BP}_{\mathrm{ND}}$ or $\mathrm{R}_{1}$ using ANOVA (region-of-interest analysis) and voxel-wise contrasts. Next, we performed linear regression models to test for potential interaction effects between age-at-onset and $\mathrm{BP}_{\mathrm{ND}} / \mathrm{R}_{1}$ on cognition. Results Both region-of-interest and voxel-wise contrasts showed higher $\left[{ }^{18} \mathrm{~F}\right]$ flortaucipir $\mathrm{BP}_{\mathrm{ND}}$ values across all neocortical regions in EOAD. By contrast, LOAD patients had lower $R_{1}$ values (indicative of more reduced $\mathrm{rCBF}$ ) in medial temporal regions. For both tau and flow in lateral temporal, and occipitoparietal regions, associations with cognitive impairment were stronger in $\mathrm{EOAD}$ than in $\mathrm{LOAD}\left(\mathrm{EOAD} \mathrm{BP} \mathrm{ND}-0.76 \leq \mathrm{st} \beta \leq-0.48\right.$ vs LOAD $-0.18 \leq \mathrm{st} \beta \leq-0.02$; EOAD $\mathrm{R}_{1}$ $0.37 \leq \mathrm{st} \beta \leq 0.84$ vs $\mathrm{LOAD}-0.25 \leq \mathrm{st} \beta \leq 0.16$ ).
\end{abstract}

Conclusions Compared to LOAD, the degree of lateral temporal and occipitoparietal tau pathology and relative cerebral blood-flow is more strongly associated with cognition in EOAD.

Keywords Early-onset $\cdot$ Alzheimer's disease $\cdot\left[{ }^{18} \mathrm{~F}\right]$ flortaucipir $\cdot$ Tau pathology $\cdot$ Cerebral blood flow $\cdot$ Cognition

\section{Introduction}

This article is part of the Topical Collection on Neurology Dementia

Denise Visser and Sander CJ Verfaillie contributed equally.

\section{Denise Visser}

d.visser2@amsterdamumc.nl

1 Department of Radiology \& Nuclear Medicine, Amsterdam Neuroscience, Vrije Universiteit Amsterdam, Amsterdam UMC, Amsterdam, Netherlands

2 Department of Neurology, Alzheimer Center Amsterdam, Amsterdam Neuroscience, Vrije Universiteit Amsterdam, Amsterdam UMC, Amsterdam, Netherlands
Alzheimer's disease (AD) is characterized by depositions of amyloid- $\beta$ plaques and hyperphosphorylated tau tangles [1]. The symptoms of $\mathrm{AD}$ are heterogeneous, partly related

3 Department of Epidemiology and Biostatistics, Vrije Universiteit Amsterdam, Amsterdam UMC, Amsterdam, Netherlands

4 Clinical Memory Research Unit, Lund University, Lund, Sweden 
to age-at-onset. Late-onset $\mathrm{AD}$ is primarily associated with amnestic problems, while non-amnestic symptoms are more prominent when the disease manifests at younger age [2-6]. Atypical cognitive profiles in early-onset AD patients have previously been linked to specific spatial patterns of hypometabolism, atrophy, and more recently, tau pathology, that primarily affect the neocortex with relative sparing of the medial temporal lobe [7-10].

Age-at-onset is thus closely related to both cognitive symptoms and distinct patterns of tau pathology in AD. However, despite the notion that tau pathology is tightly linked to the degree of cognitive impairment in AD [11-17], it is currently unknown whether the association between tau pathology and cognitive performance is affected by age-atonset. To address this question, we conducted a dynamic $\left[{ }^{18} \mathrm{~F}\right]$ flortaucipir PET study. The dynamic scan protocol additionally yields a measure of $R_{1} \cdot R_{1}$ represents the rate constant for ligand transfer from blood to tissue $\left(K_{l}\right)$ in the target region relative to the reference region, and can be interpreted as a proxy of relative cerebral blood flow [rCBF] [18-24]. Several studies have demonstrated strong correlations between $R_{1}$ and metabolic activity as measured with $\left[{ }^{18}\right.$ F]FDG PET $[19,20,24,25]$. Furthermore, in a previous study, we showed that $\left[{ }^{18} \mathrm{~F}\right]$ flortaucipir $R_{1}$ is strongly associated with cognitive performance in $\mathrm{AD}$, (partly) independently of tau pathology [17]. We therefore also investigated differences in $\mathrm{rCBF}$ and its association with cognitive performance, taking into account age-at-onset.

Accordingly, we aimed to investigate the differences between early-onset $\mathrm{AD}$ and late-onset $\mathrm{AD}$ in (1) tau pathology and $\mathrm{rCBF}$ and (2) the associations between tau pathology and rCBF with cognitive performance. We hypothesized that relative to late-onset $\mathrm{AD}$, early-onset $\mathrm{AD}$ displays higher levels of tau pathology. As $R_{1}$ is tightly linked to hypometabolism measured with FDG-PET [19, 20, 24, 25], and lower levels of metabolism have previously been reported in earlyonset $\mathrm{AD}$ patients, we expected to find lower levels of $R_{1}$ in early- vs late-onset AD. Furthermore, as early-onset AD may represent a more "pure" form of AD with relatively few comorbidities compared to late-onset AD [26], we hypothesized that tau pathology and cognitive performance would be more strongly associated in early- vs late-onset AD.

\section{Methods}

\section{Participants}

We included 79 subjects from the Amsterdam Dementia Cohort with either probable AD dementia [27] $(n=68)$ or mild cognitive impairment (MCI) due-to-AD [28] $(n=11)$, with positive amyloid- $\beta$ biomarkers [29-31]. All subjects underwent a standardized dementia screening, including medical history, extensive neuropsychological assessment, physical and neurological examination, lumbar puncture, blood tests, electroencephalography, and brain MRI. Diagnosis was established by consensus in a multidisciplinary meeting. The diagnosis of MCI/AD met core clinical criteria $[27,28]$ according to the National Institute on Aging and Alzheimer's Association (NIA$\mathrm{AA}$ ) and were biomarker supported, with an AD-like CSF profile (i.e., tau/A $\beta 42$ fraction $>0.52$ ) $[32,33]$ and/or an $\mathrm{A} \beta$-PET scan $\left(\left[{ }^{11} \mathrm{C}\right] \mathrm{PiB}\right.$ or $\left[{ }^{18} \mathrm{~F}\right]$ florbetaben $)$ showing substantial amyloid accumulation when visually assessed by an experienced nuclear medicine physician $(\mathrm{BvB})$. When both CSF and PET measures of amyloid were available, PET visual read was used to determine amyloid status. Subjects were assigned to either the early- or late-onset AD group, based on a median split (age-at-PET). Since the median age in our sample was 66 years, this led to a similar age cut-off compared to the conventional threshold of 65 years [2, 3, 6, 34]. The sample included eight patients meeting criteria for an atypical variant of $\mathrm{AD}$, including two behavioral variant $\mathrm{AD}$ (bvAD) and six posterior cortical atrophy (PCA) patients [31, 35]. All atypical $\mathrm{AD}$ patients were part of the early-onset $\mathrm{AD}$ group, except one bvAD patient. Exclusion criteria for all participants were (1) dementia not due-to-AD, (2) significant cerebrovascular disease on MRI (e.g., major cerebrovascular accident), (3) major traumatic brain injury, (4) major psychiatric or neurological disorders other than $\mathrm{AD}$, and (5) (recent) substance abuse. The study protocol was approved by the Medical Ethics Review Committee of the Amsterdam UMC, location VU Medical center. All patients provided written informed consent.

\section{$\left[{ }^{18}\right.$ F $]$ flortaucipir PET}

Acquisition and processing of $\left[{ }^{18} \mathrm{~F}\right]$ flortaucipir PET images is described in detail elsewhere [17, 25, 36]. In short, dynamic 130 -min $\left[{ }^{18} \mathrm{~F}\right]$ flortaucipir PET scans were acquired on a Philips Ingenuity TF-64 PET/CT scanner. The scanning protocol consisted of two dynamic PET scans of 60 and $50 \mathrm{~min}$, respectively, with an inbetween 20-min break [36]. The first 60-min dynamic scan started simultaneously with a bolus injection of approximately $234 \pm 14 \mathrm{MBq}\left[{ }^{18} \mathrm{~F}\right]$ flortaucipir (injected mass $1.2 \pm 0.9 \mu \mathrm{g})$. The second PET scan was co-registered to the first dynamic PET scan using Vinci software (Max Planck Institute, Germany). PET data were acquired in list mode and subsequently reconstructed using 3D-RAMLA including standard corrections for dead time, decay, attenuation, random, and scatter. Patients also underwent 3DT1-weighted MRI (Ingenuity TF PET/MR, Philips Medical Systems, The Netherlands) for anatomical and tissue segmentation purposes. 


\section{Image analysis}

The 3DT1-weighted MRI images were co-registered to their corresponding PET images using Vinci software. Anatomical regions-of-interest according to the Hammers template were subsequently delineated on the MR images and superimposed on the PET scan using PVElab [37]. Using receptor parametric mapping (RPM) with cerebellar gray matter as reference region, we generated binding potential $\left(\mathrm{BP}_{\mathrm{ND}}\right)$ maps as a measure for tau pathology. Additionally, and similar to previous work [17, 25], $R_{1}$ was generated as a proxy for rCBF [18]. Partial volume correction was applied to the PET images using Van Cittert iterative deconvolution methods (IDM), combined with highly constrained back-projections (HYPR) as described previously $[17,38,39]$. Uncorrected data are shown in the main manuscript and partial volume-corrected data are shown in the Supplementary material. In line with a previous study [17], we calculated $\mathrm{BP}_{\mathrm{ND}}$ and $\mathrm{R}_{1}$ in frontal, occipital, parietal, medial, and lateral temporal bilateral cortical lobar regions-of-interest.

In addition to the region-of-interest analyses, we performed voxel-wise analyses to (1) create average images of the different diagnostic groups (early- and late-onset AD without atypical AD cases, PCA, and bvAD), and (2) explore more fine-grained differences in $\left[{ }^{18} \mathrm{~F}\right]$ flortaucipir $\mathrm{BP}_{\mathrm{ND}}$ and $\mathrm{R}_{1}$ between early- and late-onset $\mathrm{AD}$. Therefore, we warped all native space parametric $\mathrm{BP}_{\mathrm{ND}}$ and $\mathrm{R}_{1}$ images to Montreal Neurological Institute (MNI152) space using the transformation matrixes derived from warping the coregistered T1-weighted MRI scans to MNI space using Statistical Parametric Mapping (SPM) version 12. All warped images were visually inspected for transformation errors and quality control. Average images were calculated using SPM12.

\section{Neuropsychological assessment}

We included eight neuropsychological tests $(<1$ year of PET scan), including the Dutch version of the Rey Auditory Verbal Learning Test immediate recall and delayed recall (episodic memory), Digit Span forward and backward (attention/executive functioning), Trail Making Test (TMT) version A and B (attention/executive functioning), Letter Fluency test (D-A-T) (executive functioning), and Category Fluency version animals (language) [40]. TMT-A and -B scores were inverted so that lower scores indicated worse performance. In addition, the Mini-Mental State Examination (MMSE) served as a measure of global cognition [41]. For participants who had TMT-A available but were missing TMT-B, we estimated the TMT-B by multiplying the time needed to complete the TMT-A with the mean TMT-B/A ratio from the respective diagnostic group in the respective cohort. To be included in the cognition analyses, participants were required to have at least 6 out of 9 cognitive tests available, and 72 (91\%) participants met this criterion (30 early- and 42 late-onset AD). Among the excluded cases, there was one PCA case (early-onset AD group). sFigure-1 shows the missing values in the cognitionsubsample for each neuropsychological test. There were on average $5 \%$ missing data in this subsample, for which we performed single imputation (with 5 iterations) for missing data, using all demographic (except for age and APOE4 status), neuropsychological, and neuroimaging variables as predictors $[42,43]$.

\section{Statistical analysis}

Differences in demographics and clinical characteristics between groups were assessed using independent sample $t$-tests for continuous variables and $\chi^{2}$ for dichotomous data. Differences in neuropsychological test-scores were assessed using ANOVA, adjusting for sex and education.

To assess differences in $\left[{ }^{18} \mathrm{~F}\right]$ flortaucipir $\mathrm{BP}_{\mathrm{ND}}$ and $\mathrm{R}_{1}$ between early- and late-onset $\mathrm{AD}$ groups, we used ANOVA with each of the lobar regions-of-interest (separately) as dependent variable. We repeated these analyses, but now excluding the atypical AD cases $(n=8)$, to investigate whether potential age-dependent results were not driven solely by these extreme phenotypes. To assess more fine-grained differences, we additionally performed voxel-wise contrasts in SPM12 for $\mathrm{BP}_{\mathrm{ND}}$ or $\mathrm{R}_{1}$ between early- and late-onset AD. All analyses were adjusted for sex. Results from voxel-wise analyses are displayed at both more liberal (i.e., $p<0.001$, uncorrected) and more stringent $(p<0.05$, family wise error (FWE) corrected) thresholds. Next, we investigated whether associations between $\mathrm{BP}_{\mathrm{ND}}$ or $R_{1}$ and cognition differed between early- and late-onset $\mathrm{AD}$. We performed linear regression analyses between $\mathrm{BP}_{\mathrm{ND}}$ or $R_{1}$ and neuropsychological tests (separate models), adjusted for sex and education, including the interaction term "age-at-onset (dichotomous)* $\mathrm{BP}_{\mathrm{ND}}$ or $R_{1}$ ".

Results were considered significant if $p$-values were $\leq 0.05$ for regional analyses. We considered a $p$-value $\leq 0.10$ significant for interaction terms [44]. Additionally, adjustment for multiple comparisons was performed using the Benjamini-Hochberg false discovery rate (FDR) method (indicated by $\mathrm{p}_{\mathrm{FDR}}$ ) [45]. Statistical analyses were performed using $\mathrm{R}$ software, version 4.0.2, and the "mice" package was used for imputation. 
Table 1 Demographics and cognitive test scores of the sample. Depicted are mean (SD), unless specified otherwise, for early-onset $\mathrm{AD}(\mathrm{EOAD})$ and late-onset $\mathrm{AD}$ (LOAD) groups. Median [range] is depicted for education. APOE $\varepsilon 4$ status was unknown for four LOAD subjects. Independent sample $T$-test or $\chi^{2}$ test was used for demographic variables. Differences in cognition were assessed using ANOVA, adjusted for sex and education

\begin{tabular}{llll}
\hline & EOAD & LOAD & $p$-value \\
\hline Sample size, $n$ & 35 & 44 & \\
Age-at-PET, y & $59(5)$ & $71(5)$ & $<0.001$ \\
Females, $n(\%)$ & $19(54)$ & $23(52)$ & 0.724 \\
APOE $\varepsilon 4$ carriership, $n$ & $24 / 35$ & $31 / 40$ & 0.541 \\
Education (Verhage scale*) & $6[3-7]$ & $6[3-7]$ & 0.318 \\
MMSE & $23(4)$ & $23(4)$ & 0.815 \\
Cognition & 30 & 42 & 0.013 \\
Sample size $(n)$ & $29(8)$ & $23(9)$ & 0.329 \\
RAVLT immediate recall & $3(3)$ & $2(3)$ & 0.817 \\
RAVLT delayed & $11(2)$ & $11(3)$ & 0.069 \\
Digit span forward & $7(2)$ & $7(3)$ & 0.045 \\
Digit span backward & $100(88)$ & $68(43)$ & 0.112 \\
Trail Making Test-A, sec & $334(323)$ & $244(167)$ & 0.904 \\
Trail Making Test-B, sec & $33(12)$ & $32(11)$ & 0.076 \\
Letter fluency test (D-A-T) & $17(6)$ & $14(5)$ & \\
Category fluency animals & & & \\
\hline
\end{tabular}

${ }^{*}$ The Dutch Verhage scale for education includes 7 ascending categories, ranging from one (representing less than 6 years of primary education) to 7 (representing a university degree)

\section{Results}

Participant characteristics are presented in Table 1. There were no differences in level of education, sex distribution, and APOE $\varepsilon 4$ carriership between early- and late-onset $\mathrm{AD}$ (all $p>0.05$ ), also in the cognition subsample (sTable-1). Figure 1 and sFigure-2 show average $\mathrm{BP}_{\mathrm{ND}}$ and $\mathrm{R}_{1}$ maps, respectively, to provide insight into their spatial distribution. On average, early-onset $\mathrm{AD}$ patients showed highest $\mathrm{BP}_{\mathrm{ND}}$ values in medial and lateral parietal, and lateral temporal cortical areas (Fig. 1). Late-onset $\mathrm{AD}$ patients showed highest $\mathrm{BP}_{\mathrm{ND}}$ values in lateral temporal regions, and PCA patients in parieto-occipital regions, with $\mathrm{BP}_{\mathrm{ND}}$ values exceeding all groups (Fig. 1). Lastly, bvAD patients showed highest $\mathrm{BP}_{\mathrm{ND}}$ values in lateral frontal and temporal regions, as well as lateral and medial parietal regions (Fig. 1). For $R_{1}$, values and patterns on the parametric images were visually similar across groups. (sFig-2).

\section{Early- and late-onset AD differences in $\mathrm{BP}_{\mathrm{ND}}$}

Early-onset AD patients showed higher $\left[{ }^{18} \mathrm{~F}\right]$ flortaucipir $\mathrm{BP}_{\mathrm{ND}}$ in lateral temporal $\left(\mathrm{BP}_{\mathrm{ND}} 0.56 \pm 0.30\right.$ vs $0.42 \pm 0.30$, $p=0.045)$, parietal $\left(\mathrm{BP}_{\mathrm{ND}} 0.84 \pm 0.50\right.$ vs $0.33 \pm 0.29$, $p<0.001)$, occipital $\left(\mathrm{BP}_{\mathrm{ND}} 0.65 \pm 0.52\right.$ vs $0.29 \pm 0.23$, $p<0.001)$, and frontal cortex $\left(\mathrm{BP}_{\mathrm{ND}} 0.40 \pm 0.30 \mathrm{vs}\right.$ $0.16 \pm 0.23, p<0.001$ ) compared to late-onset $\mathrm{AD}$ (Fig. 2). There were no differences in medial temporal $\mathrm{BP}_{\mathrm{ND}}$ between early-onset $\mathrm{AD}(0.24 \pm 0.14)$ and late-onset $\mathrm{AD}(0.25 \pm 0.18$, $p=0.895)$. Voxel-wise analyses confirmed higher $\mathrm{BP}_{\mathrm{ND}}$ in widespread neocortical regions in early- vs late-onset $\mathrm{AD}$ (Fig. 2G). Effects were most pronounced in the precuneus and posterior cingulate, and frontotemporal cortex, as supported by the FWE-corrected results. There were no regions in which late-onset $\mathrm{AD}$ showed higher $\mathrm{BP}_{\mathrm{ND}}$ compared to early-onset AD (Fig. 2G). Results remained essentially unchanged when PCA and bvAD patients were excluded from the analysis (sFig-3). Furthermore, results from partial volume corrected data yielded highly similar results, although $\mathrm{BP}_{\mathrm{ND}}$ values were slightly higher (sFig-4).

\section{Early- and late-onset AD differences in $\mathbf{R}_{\mathbf{1}}$}

By contrast, $\left[{ }^{18} \mathrm{~F}\right]$ flortaucipir $R_{1}$ was lower in late-onset $\mathrm{AD}$ compared to early-onset $\mathrm{AD}$ in the medial temporal lobe $\left(R_{1} 0.66 \pm 0.05\right.$ vs $\left.0.69 \pm 0.05, p<0.05\right)$, but not in any of the neocortical regions ( $p>0.05)$ (Fig. 3). Voxelwise analyses showed lower $R_{1}$ in the (medial) temporal lobe (surviving FWE-correction), and subtly lower $R_{1}$ in the medial frontal cortex in late-onset compared to early-onset AD (Fig. 3G). In contrast, parieto-occipital regions showed lower $R_{1}$ in early-onset $\mathrm{AD}$ compared to late-onset $\mathrm{AD}$, but this did not survive FWE-correction. Results remained essentially unchanged when atypical cases were excluded from the analysis (sFig-5). Results from partial volume corrected data yielded highly similar results, although $R_{1}$ values were slightly higher (sFig-6).

\section{Early- and late-onset AD differences in the association between $\mathrm{BP}_{\mathrm{ND}}$ and cognition}

Linear regression analyses showed that, in general, higher $\left[{ }^{18} \mathrm{~F}\right]$ flortaucipir $\mathrm{BP}_{\mathrm{ND}}$ was strongly associated with worse scores on a variety of cognitive tests (Fig. 4A). Age-at-onset moderated these associations such that in early-onset $\mathrm{AD}$, associations between lateral temporal, parietal, and occipital $\mathrm{BP}_{\mathrm{ND}}$ with TMT-A, and parietal $\mathrm{BP}_{\mathrm{ND}}$ with TMT-B were stronger than in late-onset $\mathrm{AD}$ (all $p_{\text {interaction }}<0.10$ : Fig. 4). In late-onset $\mathrm{AD}$, associations between medial temporal $\mathrm{BP}_{\mathrm{ND}}$ and Digit Span backward and Letter fluency test (D-A-T) were stronger than in early-onset AD (Fig. 4). Results from partial volume corrected data were essentially comparable (sFig-7). 


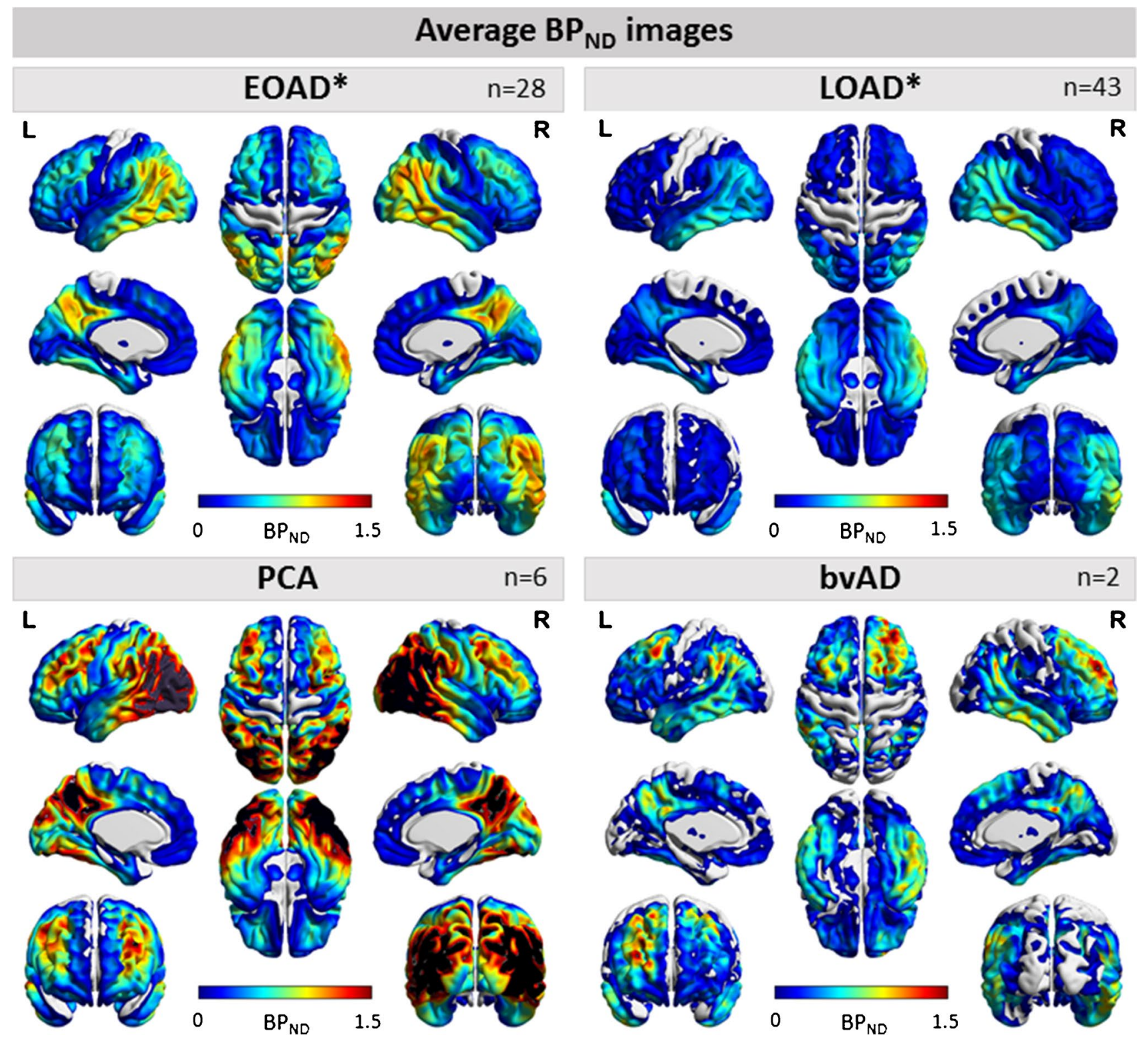

Fig. 1 Average $\left[{ }^{18} \mathrm{~F}\right]$ flortaucipir $\mathrm{BP}_{\mathrm{ND}}$ images for early- and late-onset $\mathrm{AD}, \mathrm{PCA}$, and bvAD. Average images of all early-onset Alzheimer's disease (EOAD), late-onset AD (LOAD), posterior cortical atrophy (PCA) patients, and behavioral variant $\mathrm{AD}$ (bvAD) patients on a scale

\section{Early- and late-onset AD differences in the association between $R_{1}$ and cognition}

In general, lower $\left[{ }^{18} \mathrm{~F}\right]$ flortaucipir $R_{1}$ was associated with worse scores on multiple cognitive tests (Fig. 5). Age-at-onset moderated these associations such that in early-onset $\mathrm{AD}$, associations were stronger than in late-onset $\mathrm{AD}$ (all $p_{\text {interaction }}<0.10$ : Fig. 5). More specifically, this was the case for the association between lateral temporal $R_{1}$ and Digit Span backward, TMTA, TMT-B, and Letter fluency test (D-A-T), and the associations between occipito-parietal $\mathrm{R}_{1}$ and TMT-A, TMT-B, and ranging from $\mathrm{BP}_{\mathrm{ND}} 0$ to 1.5 . *Excluding atypical variants, posterior cortical atrophy (PCA) patients and behavioral variant $\mathrm{AD}$ (bvAD) patients

Category fluency Animals, as well as the association between occipital $\mathrm{R}_{1}$ and MMSE (Fig. 5). Results from partial volume corrected data yielded some (test-specific) differences compared to the results from the uncorrected PET data (sFig-8).

\section{Discussion}

The main findings of this study include higher levels of neocortical tau pathology in early-onset AD compared to late-onset $\mathrm{AD}$, while late-onset $\mathrm{AD}$ showed reduced $\mathrm{rCBF}$ 

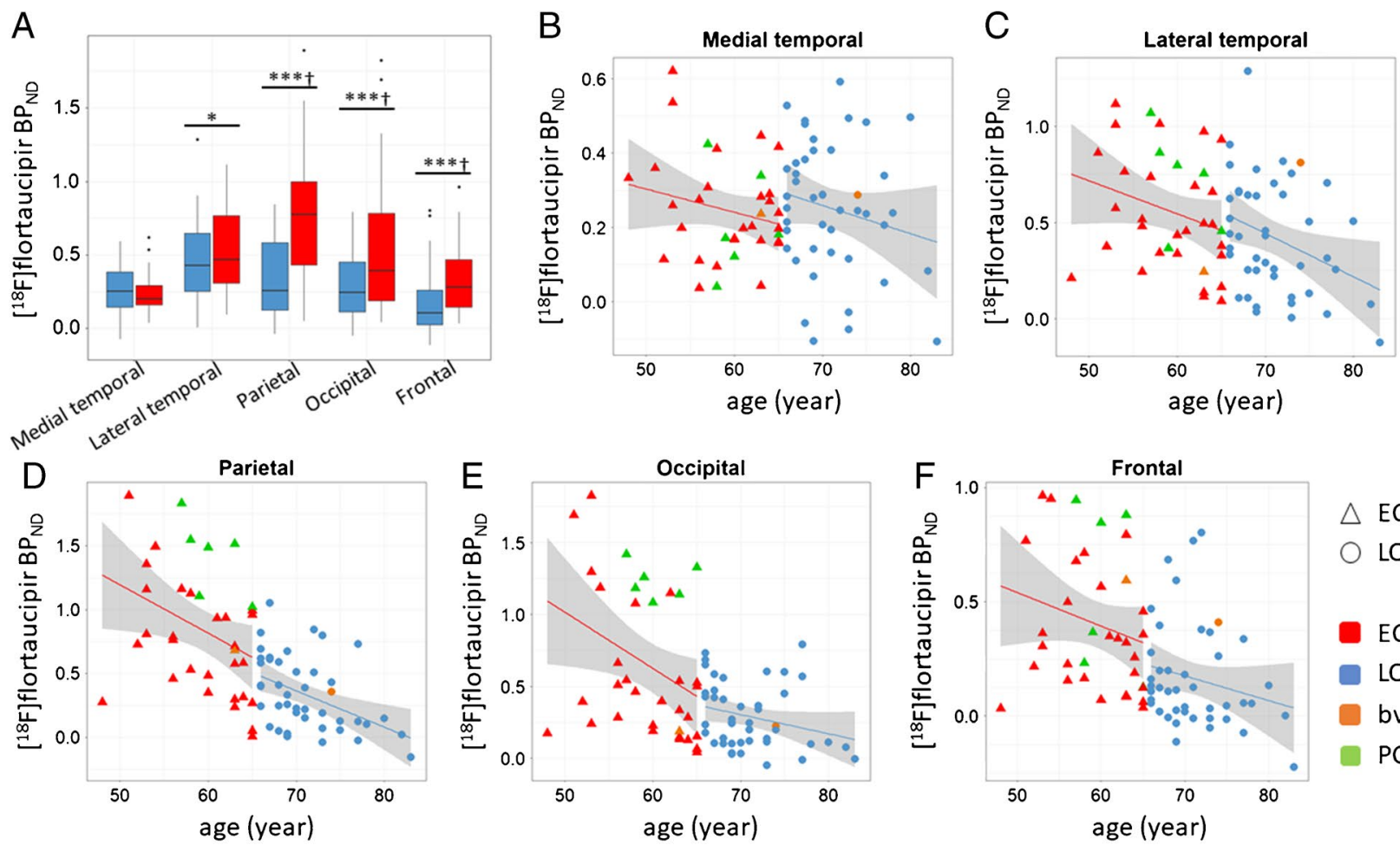

$\triangle E O A D$

O LOAD

G

$\left[{ }^{18} \mathrm{~F}\right]$ flortaucipir $\mathrm{BP} \mathrm{ND}_{\mathrm{N}}$

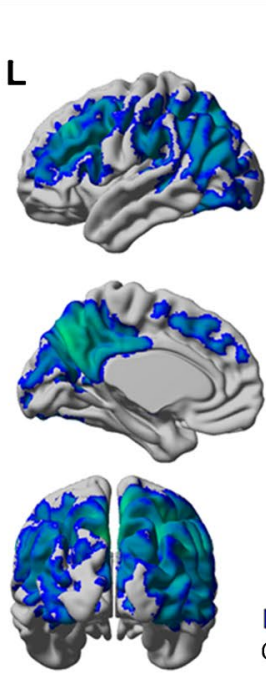

EOAD > LOAD
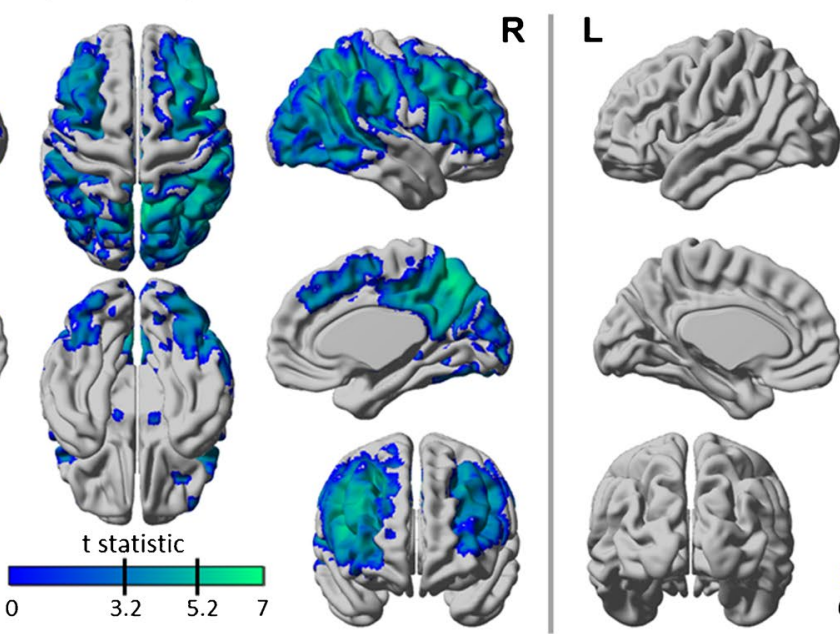

\section{EOAD $<$ LOAD}

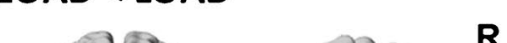

EOAD

LOAD

bVAD

PCA
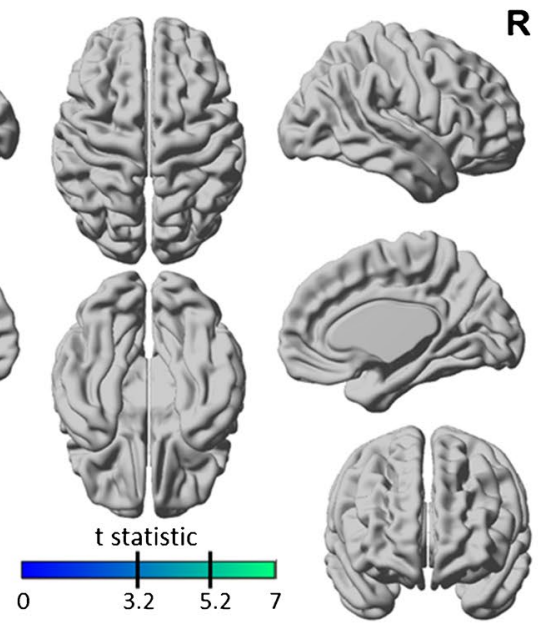

Fig. $2\left[{ }^{18} \mathrm{~F}\right]$ flortaucipir $\mathrm{BP}_{\mathrm{ND}}$ for early- and late-onset AD. A Boxplot of $\left[{ }^{18} \mathrm{~F}\right]$ flortaucipir $\mathrm{BP}_{\mathrm{ND}}$ values for each region-of-interest (ROI). Differences were assessed using ANOVA, adjusted for sex. B-F Scatterplots for $\left[{ }^{18} \mathrm{~F}\right]$ flortaucipir $\mathrm{BP}_{\mathrm{ND}}$ by age for each ROI. $\mathbf{G}$ Results from voxel-wise contrast for $\left[{ }^{18} \mathrm{~F}\right]$ flortaucipir $\mathrm{BP}_{\mathrm{ND}}$ between

early-onset $\mathrm{AD}$ (EOAD) and late-onset $\mathrm{AD}$ (LOAD). Indicated by the black lines on the color scale are thresholds for $p<0.001$, uncorrected $(t=3.20)$ and for $p<0.05$, FWE-corrected $(t=5.24) *{ }^{*} p<0.05$; $* * p<0.01 ; * * * p<0.001 ; \dagger \mathrm{p}_{\mathrm{FDR}}<0.05$

in the medial temporal lobe compared to early-onset AD. Moreover, we found that higher levels of tau pathology and lower cerebral blood flow in lateral temporal and occipitoparietal regions were more strongly associated with cognitive impairment (mainly executive functioning domain) in early- vs late-onset AD. Previous studies demonstrated that

relative to late-onset $\mathrm{AD}$, early-onset $\mathrm{AD}$ exhibits more extensive pathological and neurodegenerative changes with respect to (among others) amyloid, metabolic activity [7, 46], atrophy [47, 48], functional network changes [49-51], and tau [52, 53]. The current study extends on these previous studies not only showing greater tau load in 

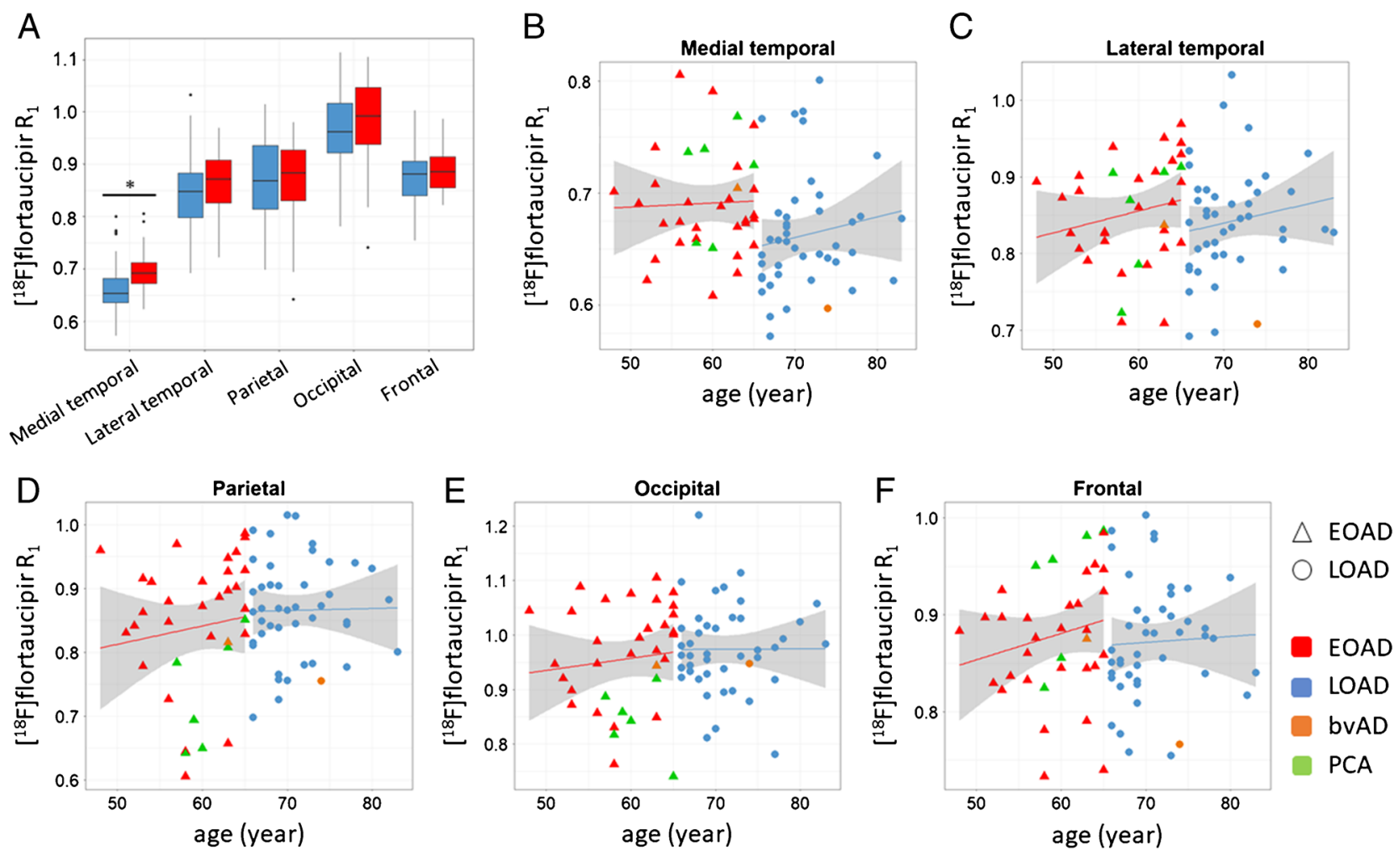

G

$\left[{ }^{18} \mathrm{~F}\right]$ flortaucipir $\mathbf{R}_{\mathbf{1}}$

EOAD > LOAD

$\mathbf{L}$
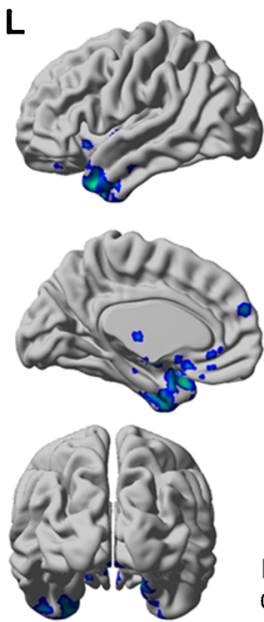
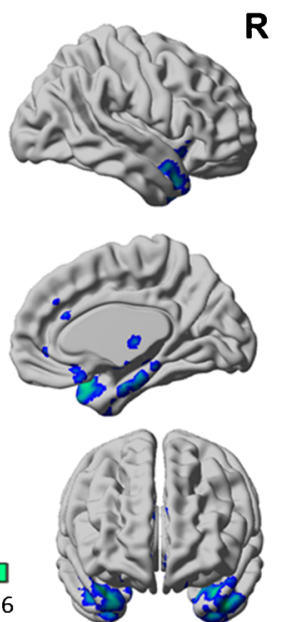

$\mathbf{R}$

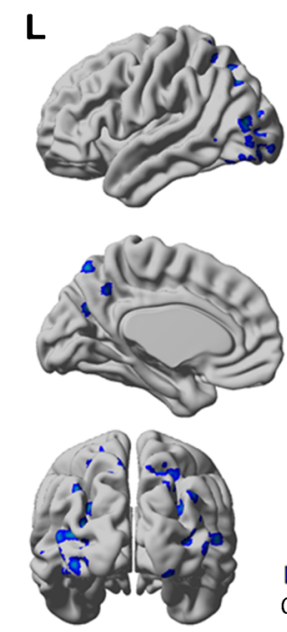

EOAD $<$ LOAD
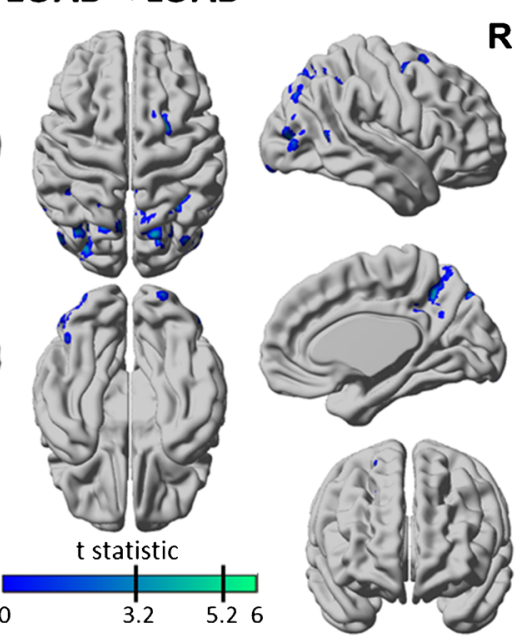

Fig. $3\left[{ }^{18} \mathrm{~F}\right]$ flortaucipir $\mathrm{R}_{1}$ for early- and late-onset AD. A Boxplot of $\left[{ }^{18} \mathrm{~F}\right]$ flortaucipir $R_{1}$ values for each region-of-interest (ROI). B-F Scatterplots for $\left[{ }^{18} \mathrm{~F}\right]$ flortaucipir $\mathrm{R}_{1}$ by age for each ROI. G Results from voxel-wise contrast for $\left[{ }^{18} \mathrm{~F}\right]$ flortaucipir $\mathrm{R}_{1}$ between early-onset

early- vs late-onset $\mathrm{AD}$, but also providing novel insights into age-dependent differences in cerebral blood flow and demonstrates differential associations of both biomarkers with cognition. These findings contribute to our understanding of the differences between early- and late-onset $\mathrm{AD}$ patients and may support that younger AD patients are
$\mathrm{AD}(\mathrm{EOAD})$ and late-onset $\mathrm{AD}(\mathrm{LOAD})$. Indicated by the black lines on the color scale are thresholds for $p<0.001$, uncorrected $(t=3.20)$ and for $p<0.05$, FWE-corrected $(t=5.24)$. $* p<0.05$

more suitable for inclusion in clinical trials, as the stronger link between pathology and cognitive decline suggests that a greater benefit may be achieved in this population when targeting tau pathology or cerebral blood flow compared to older AD patients, where this link is less strong. Besides, our results emphasize the importance of cerebrovascular 
A
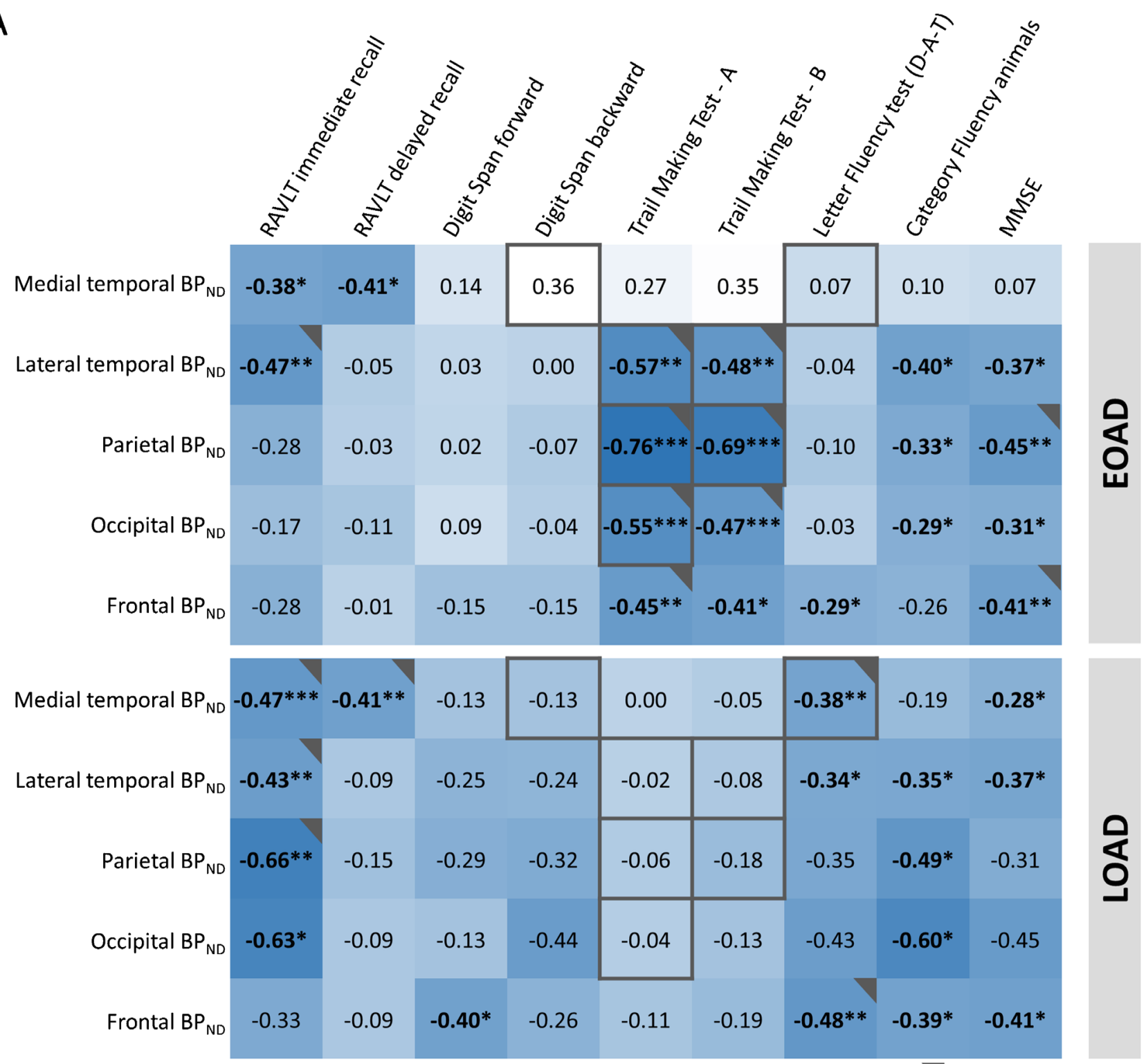

B

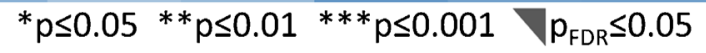

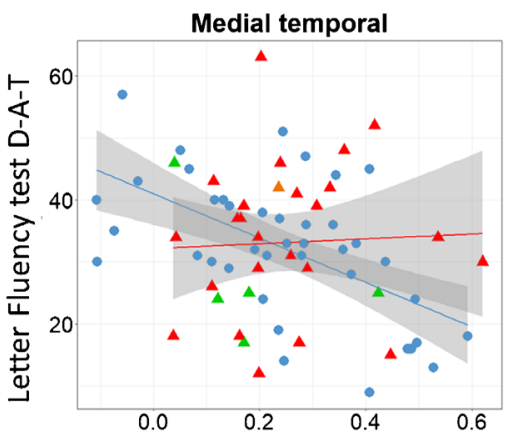

$\left[{ }^{18} \mathrm{~F}\right]$ flortaucipir $\mathrm{BP}_{\mathrm{ND}}$

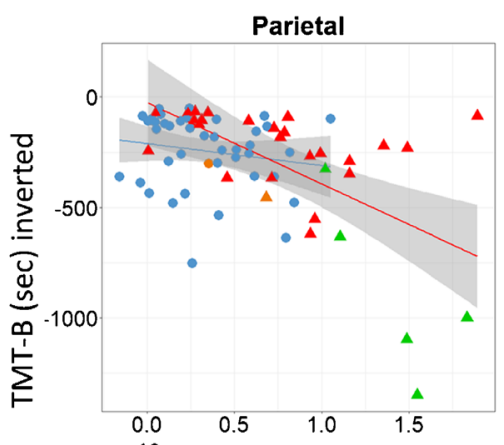

$\left[{ }^{18} \mathrm{~F}\right]$ flortaucipir $B P_{N D}$

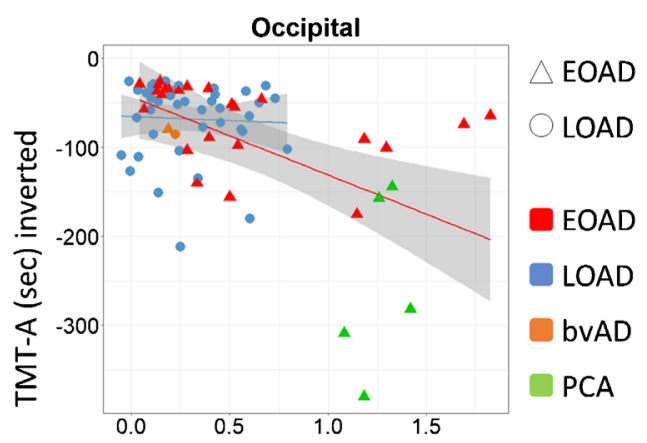

$\left[{ }^{18} \mathrm{~F}\right]$ flortaucipir $\mathrm{BP}_{\mathrm{ND}}$
Fig. 4 Associations between $\left[{ }^{18} \mathrm{~F}\right]$ flortaucipir $\mathrm{BP}_{\mathrm{ND}}$ and cognitive test scores for early- and late-onset AD. A Significant modification of age-at-onset as assessed in the model including all AD subjects from the cognition subsample $(n=72)$, adjusted for age, sex, and education is depicted in grey (representing interaction terms at $p \leq 0.10$ ) and black (representing interaction terms at $p_{\mathrm{FDR}} \leq 0.05$ ) squares. Standardized regression coefficients are depicted for early-onset AD (EOAD) and late-onset AD (LOAD) separately. B Selection of scatterplots for the association between $\left[{ }^{18} \mathrm{~F}\right]$ flortaucipir $\mathrm{BP}_{\mathrm{ND}}$ and neuropsychological test scores 
A

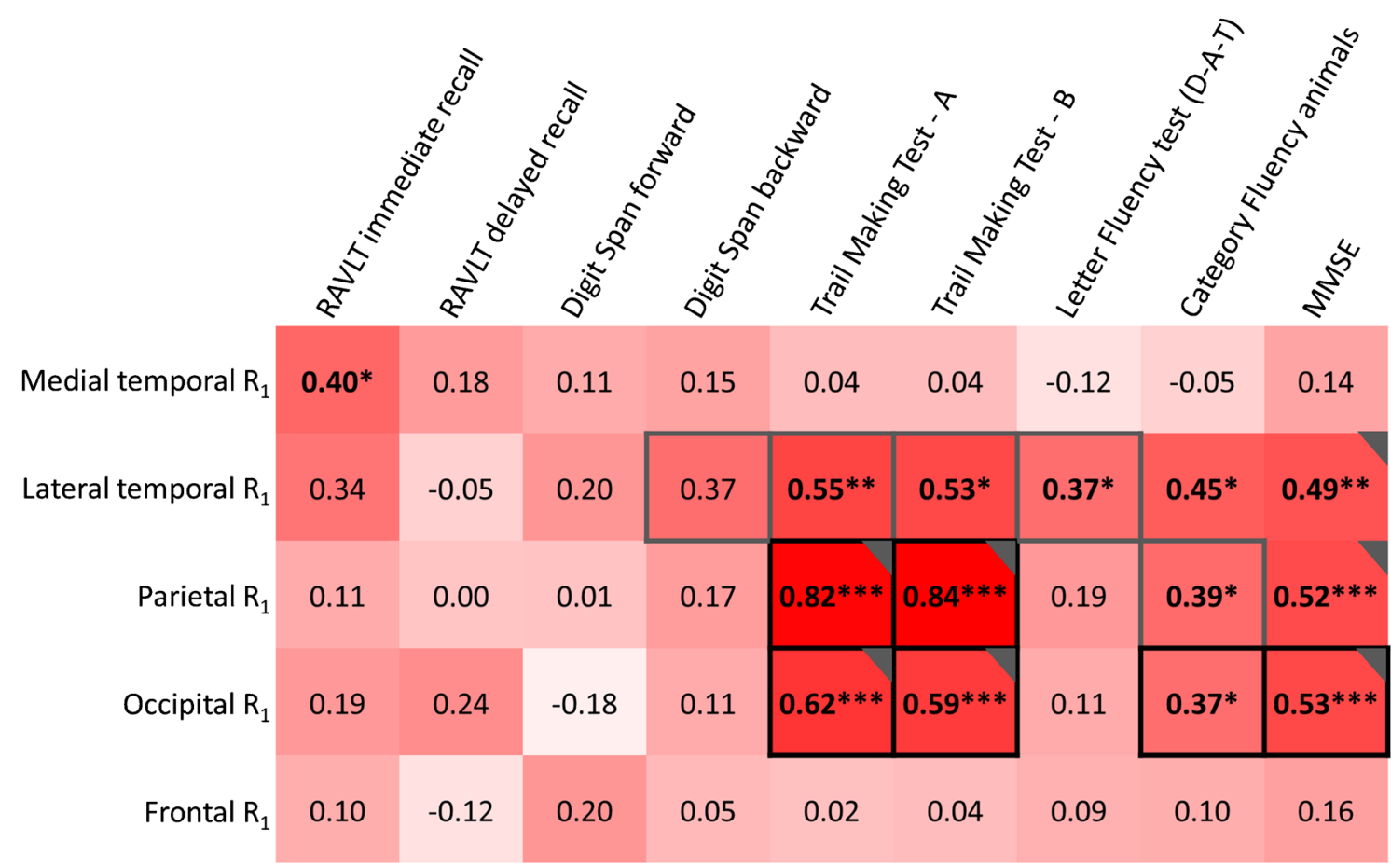

\begin{tabular}{|c|c|c|c|c|c|c|c|c|c|}
\hline Medial temporal $R_{1}$ & 0.15 & 0.04 & 0.17 & -0.17 & -0.01 & -0.06 & -0.02 & 0.01 & -0.02 \\
\hline Lateral temporal $\mathrm{R}_{1}$ & 0.22 & 0.06 & 0.03 & -0.10 & 0.06 & 0.04 & -0.04 & 0.04 & 0.17 \\
\hline Parietal $R_{1}$ & 0.19 & 0.03 & 0.01 & -0.01 & 0.16 & 0.14 & -0.02 & -0.02 & 0.22 \\
\hline Occipital $R_{1}$ & -0.10 & -0.10 & -0.15 & -0.16 & -0.02 & -0.04 & -0.19 & -0.25 & -0.08 \\
\hline Frontal $\mathrm{R}_{1}$ & 0.17 & 0.12 & 0.19 & -0.10 & 0.08 & 0.02 & 0.02 & 0.06 & 0.16 \\
\hline
\end{tabular}

B

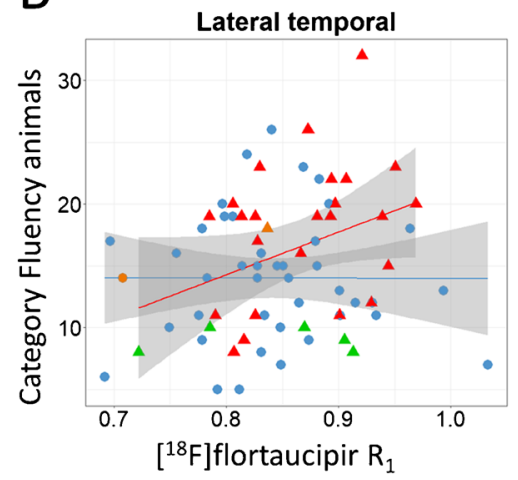

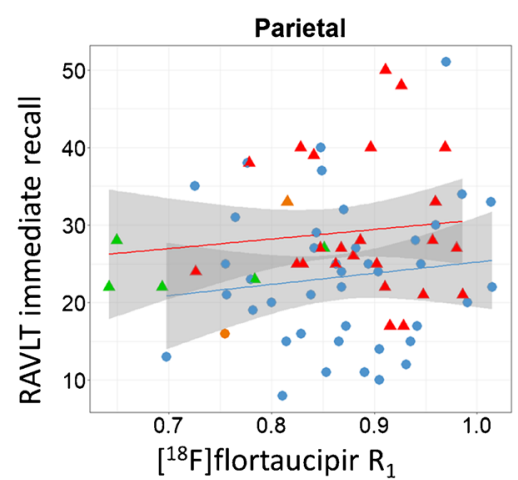

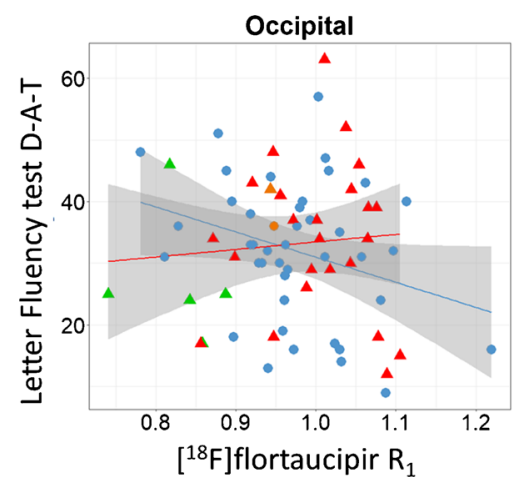

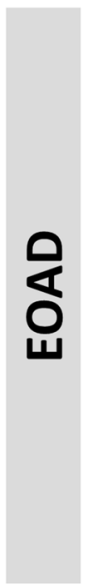

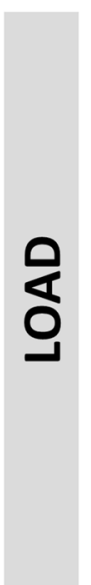

$\triangle \mathrm{EOAD}$

LOAD

DEAD

LOAD

bvAD

PCA
Fig. 5 Associations between $\left[{ }^{18} \mathrm{~F}\right]$ flortaucipir $R_{1}$ and cognitive test scores for early- and late-onset AD. A Significant modification of age-at-onset as assessed in the model including all $\mathrm{AD}$ subjects from the cognition subsample $(n=72)$, adjusted for age, sex, and education is depicted in grey (representing interaction terms at $p \leq 0.10$ ) and black (representing interaction terms at $p_{\mathrm{FDR}} \leq 0.05$ ) squares. Standardized regression coefficients are depicted for early- and late-onset AD separately. B Selection of scatterplots for the association between $\left[{ }^{18} \mathrm{~F}\right]$ flortaucipir $\mathrm{R}_{1}$ and neuropsychological test scores 
health (and its potential treatment) in younger AD patients specifically.

For tau pathology, associations with worse cognitive performance across non-memory domains were stronger in early-onset $\mathrm{AD}$, apart from associations in the medial temporal lobe. The medial temporal region is predominantly involved in sporadic (late-onset) AD [16, 51, 54], and in line with this, associations with cognitive performance for this region were stronger in late-onset $\mathrm{AD}$. For $\mathrm{rCBF}$, we found that lower blood flow was associated with a higher degree of cognitive impairment in non-memory domains in early- vs late-onset AD. This indicates that higher levels of neocortical tau pathology and lower rCBF have a relatively stronger influence on cognitive (dys)functioning in early-onset $\mathrm{AD}$. The main hypothesis for these findings is that co-pathologies (e.g., other proteinopathies such as TDP-43 or alpha-synuclein, or vascular damage) often developing at older age, may contribute to progressive cognitive impairment in late-onset AD [55-57]. However, a recent study showed that co-pathologies also play an important role in the clinical phenotype of early-onset AD [26]. Another explanation could be that the brain regions most heavily affected in early-onset AD are more important for broader cognitive functioning (other than memory-specific), and comprise specific neuronal networks crucial for specific cognitive functions. The parietal lobe contains a high pathologic burden in early-onset AD (as shown by the result of the current study and by others (e.g., $[7,51,53])$ ), and is an important hub in higherorder cognitive networks [58]. If networks like these, or their cross-network relationships, segregate with older age [59-61], it might be that the relative importance of the regions affected by either tau pathology or decreases in rCBF decrease with older age. This would be reflected by a weakened association between tau pathology or rCBF and cognition (executive functioning specifically) in latecompared to early-onset AD.

In the current study, we used $R_{1}$ images as a measure of cerebral blood flow. This measure is tightly linked to hypometabolism measured with FDG-PET [19, 24], and others have shown decreased occipitoparietal glucose metabolism in early-onset $\mathrm{AD}[7,62]$. In contrast, our regional analyses did not show lower rCBF in early- vs late-onset AD. However, additional voxel-wise comparisons showed more fine-grained decreased $\mathrm{rCBF}$ in lateral parietal and occipital regions in early-onset $\mathrm{AD}$. One explanation for these findings might be that there are no extensive or clear differential patterns of cortical reductions in rCBF in early- vs late-onset $\mathrm{AD}$, or that differential reductions in $\mathrm{rCBF}$ may be restricted to specific cortical gyri. Another explanation could be that $R_{1}$, which is a proxy of $\mathrm{rCBF}$, is not sensitive enough to capture the differences between early- and late-onset AD. As rCBF decreases with age [63], this might suggest that early-onset $\mathrm{AD}$ patients are more severely affected by deficits in $\mathrm{rCBF}$ compared to late-onset $\mathrm{AD}$ patients, which in turn might be caused (in part) by the higher levels of tau pathology present. Ideally, one would investigate whether early-onset $\mathrm{AD}$ patients are indeed more heavily affected by reduced $\mathrm{rCBF}$ by including age-matched control groups, but since we had no such data available, it remains to be elucidated in future research.

Our results showed that early-onset AD patients exhibit higher levels of tau pathology in widespread neocortical regions compared to late-onset $\mathrm{AD}$ patients, but no differences were found in the medial temporal lobe. Others found higher levels of tau pathology primarily in (pre)frontal and (inferior) parietal cortices in early- relative to late-onset $\mathrm{AD}$, and no differences in the medial temporal cortex [53]. Another study similarly showed that early-onset AD patients showed greater binding in the inferior parietal, occipital, and inferior temporal cortices [64]. The pattern of cortical involvement as found in the current study thus is highly consistent with previous findings, indicating that the development of high levels of tau pathology follows a specific spatial pattern within early-onset AD patients. Supporting this, a recent study identified four distinct spatiotemporal trajectories of tau pathology in $\mathrm{AD}$, each presenting with distinct demographic and cognitive profiles and differing longitudinal outcomes. The medial temporal lobe-sparing subtype, for example, was associated with younger age, less APOE4 allele carriership, and greater overall tau burden [65], which is largely in accordance with findings in our early-onset $\mathrm{AD}$ study population. Others point toward genetics explaining higher tau burden, since comparable results were found in autosomal dominant mutation carriers (e.g., presenilin-1) $[66,67]$. The differential spatial patterns of tau pathology in early-onset $\mathrm{AD}$ might also be explained by genetic involvement, as early-onset AD patients are less frequently APOE4 allele carriers, and $\mathrm{E} 4$ genotype does influence spatial patterns of brain pathology [68-70]. In the present study, however, we did find slightly but non-significantly lower E4 allele carriership in early-onset $\mathrm{AD}$, which makes it unlikely that E4 genotype may have explained the findings.

A strength of this study is usage of a single dynamic $\left[{ }^{18} \mathrm{~F}\right]$ flortaucipir PET scan to derive measures of both tau pathology $\left(\mathrm{BP}_{\mathrm{ND}}\right)$ and relative cerebral blood flow $\left(R_{1}\right)$. Furthermore, we repeated analyses with partial volumecorrected data. Although we did find some (test-specific) differences in the association between $R_{1}$ and cognition, core results that led to our conclusions remained. Given that the implications of applying partial volume-correction to $R_{1}$ data are currently not entirely understood, and that all other results remained essentially comparable, we feel atrophy does not bias our findings to a large extent. There were also several limitations. First, the late-onset AD patients in our study were relatively young, which might 
hamper the generalizability of results to older study populations. Second, seven participants did not have sufficient neuropsychological data available for cognition analyses. Although results are not expected to be influenced to a large extent, it might be that results as described in this study are slightly underestimating the differential effect of age-at-onset, given that neuropsychological data were missing in the most severely progressed patients (mean MMSE score: $20 \pm 4$ ). Third, the cross-sectional design did not allow investigating the temporal evolution of tau pathology, rCBF, and cognitive impairment. Future studies with longitudinal data available could aid in elucidating the timely pathways of these three parameters.

In conclusion, early-onset $\mathrm{AD}$ is characterized by higher levels of tau pathology and stronger associations between lateral temporal and occipitoparietal tau pathology or lower rCBF and cognitive impairment. These findings may have important implications for clinical trials, since effects of potential tau- or blood flow targeting therapeutic interventions might exert larger effects in early-onset AD compared to late-onset $\mathrm{AD}$ patients. Furthermore, differences between early- and late-onset AD patients as described in the current study should be considered in any therapeutic intervention trial where either cognition is used as an outcome measure or where indirect effects on tau pathology or rCBF are expected, to ensure correct interpretation of results and aid in the formation of appropriate patient selection criteria.

Supplementary Information The online version contains supplementary material available at https://doi.org/10.1007/s00259-021-05669-6.

Acknowledgements We kindly thank all participants for their contribution. Research of Amsterdam Alzheimer Center is part of the Neurodegeneration program of Amsterdam Neuroscience. The Amsterdam Alzheimer Center is supported by Alzheimer Nederland and Stichting VUmc funds. $\left[{ }^{18} \mathrm{~F}\right]$ Flortaucipir PET scans were made possible by Avid Radiopharmaceuticals Inc.

Funding This study was funded by a ZonMW Memorabel grant. ZonMw,Memorabel Grant,Bart NM van Berckel

\section{Declarations}

Ethics approval All procedures performed in studies involving human participants were in accordance with the ethical standards of the institutional and/or national research committee and with the 1964 Helsinki declaration and its later amendments or comparable ethical standards.

Informed consent Informed consent was obtained from all individual participants included in the study.

Conflict of interest Visser, Verfaillie, Wolters, Coomans, Timmers, Tuncel, Boellaard, Golla, Windhorst, and Ossenkoppele declare that he/she has no conflict of interest.

Van der Flier received grant support from ZonMW, NWO, EU-FP7, Alzheimer Nederland, CardioVascular Onderzoek Nederland, Stichting Dioraphte, Gieskes-Strijbis Fonds, Boehringer Ingelheim, Piramal
Neuroimaging, Roche BV, Janssen Stellar, Combinostics. All the funding is paid to the institution. WvdF holds the Pasman chair.

Van Berckel received research support from ZonMW, AVID radiopharmaceuticals, CTMM, and Janssen Pharmaceuticals. He is a trainer for Piramal and GE. He receives no personal honoraria.

Scheltens received grant support (to the institution) from GE Healthcare, Danone Research, Piramal, and MERCK. In the past 2 years, he has received consultancy/speaker fees from Lilly, GE Healthcare, Novartis, Forum, Sanofi, Nutricia, Probiodrug, and EIP Pharma. All the funding is paid to the institution.

No other potential conflicts of interest relevant to this article exist.

Open Access This article is licensed under a Creative Commons Attribution 4.0 International License, which permits use, sharing, adaptation, distribution and reproduction in any medium or format, as long as you give appropriate credit to the original author(s) and the source, provide a link to the Creative Commons licence, and indicate if changes were made. The images or other third party material in this article are included in the article's Creative Commons licence, unless indicated otherwise in a credit line to the material. If material is not included in the article's Creative Commons licence and your intended use is not permitted by statutory regulation or exceeds the permitted use, you will need to obtain permission directly from the copyright holder. To view a copy of this licence, visit http://creativecommons.org/licenses/by/4.0/.

\section{References}

1. Jack CR Jr, et al. NIA-AA research framework: toward a biological definition of Alzheimer's disease. Alzheimers Dement. 2018;14(4):535-62.

2. Koedam EL, et al. Early-versus late-onset Alzheimer's disease: more than age alone. J Alzheimers Dis. 2010;19(4):1401-8.

3. Koedam EL, et al. Early-onset dementia is associated with higher mortality. Dement Geriatr Cogn Disord. 2008;26(2):147-52.

4. Koss E, et al. Clinical and neuropsychological differences between patients with earlier and later onset of Alzheimer's disease: a CERAD analysis. Part XII Neurology. 1996;46(1):136-41.

5. Scheltens NM, et al. The identification of cognitive subtypes in Alzheimer's disease dementia using latent class analysis. J Neurol Neurosurg Psychiatry. 2016;87(3):235-43.

6. Smits LL, et al. Early onset Alzheimer's disease is associated with a distinct neuropsychological profile. J Alzheimers Dis. 2012;30(1):101-8.

7. Ossenkoppele R, et al. Amyloid burden and metabolic function in early-onset Alzheimer's disease: parietal lobe involvement. Brain. 2012;135(7):2115-25.

8. Smits LL, et al. Regional atrophy is associated with impairment in distinct cognitive domains in Alzheimer's disease. Alzheimers Dement. 2014;10:S299-305.

9. Hsu J-L, et al. Posterior atrophy and medial temporal atrophy scores are associated with different symptoms in patients with Alzheimer's disease and mild cognitive impairment. PLoS ONE. 2015;10(9):e0137121.

10. Whitwell JL, et al. Neuroimaging correlates of pathologically defined subtypes of Alzheimer's disease: a case-control study. The Lancet Neurology. 2012;11(10):868-77.

11. Pontecorvo MJ, et al. Relationships between flortaucipir PET tau binding and amyloid burden, clinical diagnosis, age and cognition. Brain. 2017;140(3):748-63.

12. Aschenbrenner AJ, et al. Influence of tau PET, amyloid PET, and hippocampal volume on cognition in Alzheimer disease. Neurology. 2018;91(9):e859-66. 
13. Johnson KA, et al. Tau positron emission tomographic imaging in aging and early Alzheimer disease. Ann Neurol. 2016;79(1):110-9.

14. Ossenkoppele R, et al. Tau PET patterns mirror clinical and neuroanatomical variability in Alzheimer's disease. Brain. 2016;139(5):1551-67.

15. Ossenkoppele $\mathrm{R}$, et al. Associations between tau, $A \beta$, and cortical thickness with cognition in Alzheimer disease. Neurology. 2019;92(6):e601-12.

16. Schöll M, et al. PET imaging of tau deposition in the aging human brain. Neuron. 2016;89(5):971-82.

17. Visser $\mathrm{D}$, et al. Tau pathology and relative cerebral blood flow are independently associated with cognition in Alzheimer's disease. Eur J Nucl Med Mol Imaging. 2020;47:3165-75.

18. Golla SS, et al. Parametric methods for [18F] flortaucipir PET. J Cereb Blood Flow Metab. 2020;40(2):365-73.

19. Peretti, D.E., et al. (2019) Relative cerebral flow from dynamic PIB scans as an alternative for FDG scans in Alzheimer's disease PET studies. PloS one 14(1) e0211000

20. Rodriguez-Vieitez E, et al. Comparability of [18F] THK5317 and [11C] PIB blood flow proxy images with [18F] FDG positron emission tomography in Alzheimer's disease. J Cereb Blood Flow Metab. 2017;37(2):740-9.

21. Peretti DE, et al. Diagnostic performance of regional cerebral blood flow images derived from dynamic PIB scans in Alzheimer's disease. EJNMMI Res. 2019;9(1):1-9.

22. Joseph-Mathurin N, et al. Utility of perfusion PET measures to assess neuronal injury in Alzheimer's disease. Alzheimer's \& Dementia: Diagnosis, Assessment \& Disease Monitoring. 2018;10:669-77.

23. Chen YJ, et al. Relative $11 \mathrm{C}-\mathrm{PiB}$ delivery as a proxy of relative CBF: quantitative evaluation using single-session 15O-water and 11C-PiB PET. J Nucl Med. 2015;56(8):1199-205.

24. Ottoy J, et al. 18F-FDG PET, the early phases and the delivery rate of $18 \mathrm{~F}-\mathrm{AV} 45 \mathrm{PET}$ as proxies of cerebral blood flow in Alzheimer's disease: Validation against 15O-H2O PET. Alzheimers Dement. 2019;15(9):1172-82.

25. Wolters EE, et al. Tau pathology, relative cerebral flow and cognition in dementia with Lewy bodies: Tau imaging. Alzheimers Dement. 2020;16:e041048.

26. Spina, S., et al., 2021 Comorbid neuropathological diagnoses in early versus late-onset Alzheimer's disease. Brain,

27. McKhann GM, et al. The diagnosis of dementia due to Alzheimer's disease: recommendations from the National Institute on Aging-Alzheimer's Association workgroups on diagnostic guidelines for Alzheimer's disease. Alzheimers Dement. 2011;7(3):263-9.

28. Albert MS, et al. The diagnosis of mild cognitive impairment due to Alzheimer's disease: recommendations from the National Institute on Aging-Alzheimer's Association workgroups on diagnostic guidelines for Alzheimer's disease. Focus. 2013;11(1):96-106.

29. van der Flier WM, et al. Optimizing patient care and research: the Amsterdam Dementia Cohort. J Alzheimers Dis. 2014;41(1):313-27.

30. Crutch SJ, et al. Posterior cortical atrophy. The Lancet Neurology. 2012;11(2):170-8.

31. Ossenkoppele R, et al. The behavioural/dysexecutive variant of Alzheimer's disease: clinical, neuroimaging and pathological features. Brain. 2015;138(9):2732-49.

32. Tijms BM, et al. Unbiased approach to counteract upward drift in cerebrospinal fluid amyloid- $\beta$ 1-42 analysis results. Clin Chem. 2018;64(3):576-85.

33. Duits FH, et al. The cerebrospinal fluid "Alzheimer profile": easily said, but what does it mean? Alzheimers Dement. 2014;10(6):713-23.
34. Mendez MF, et al. Nonamnestic presentations of early-onset Alzheimer's disease. Am J Alzheimers Dis Other Demen. 2012;27(6):413-20.

35. Crutch SJ, et al. Consensus classification of posterior cortical atrophy. Alzheimers Dement. 2017;13(8):870-84.

36. Golla SS, et al. Quantification of tau load using [18 F] AV1451 PET. Mol Imag Biol. 2017;19(6):963-71.

37. Rask, T., et al., 2004 PVElab: software for correction of functional images for partial volume errors. Neuroimage, 22

38. Golla SS, et al. Partial volume correction of brain PET studies using iterative deconvolution in combination with HYPR denoising. EJNMMI Res. 2017;7(1):1-12.

39. Cysouw M, et al. Partial-volume correction in dynamic PET-CT: effect on tumor kinetic parameter estimation and validation of simplified metrics. EJNMMI Res. 2019;9(1):1-11.

40. Groot $\mathrm{C}$, et al. Differential effects of cognitive reserve and brain reserve on cognition in Alzheimer disease. Neurology. 2018;90(2):e149-56.

41. Folstein MF, Folstein SE, McHugh PR. "Mini-mental state": a practical method for grading the cognitive state of patients for the clinician. J Psychiatr Res. 1975;12(3):189-98.

42. Rubin, D.B., 2004 Multiple imputation for nonresponse in surveys. Vol. 81 John Wiley \& Sons.

43. Von Hippel PT. How many imputations do you need? A twostage calculation using a quadratic rule. Sociological Methods \& Research. 2020;49(3):699-718.

44. Selvin, S., 2004 Statistical analysis of epidemiologic data. Oxford University Press.

45. Benjamini Y, Hochberg Y. Controlling the false discovery rate: a practical and powerful approach to multiple testing. J Roy Stat Soc: Ser B (Methodol). 1995;57(1):289-300.

46. Lehmann M, et al. Diverging patterns of amyloid deposition and hypometabolism in clinical variants of probable Alzheimer's disease. Brain. 2013;136(3):844-58.

47. Chan D, et al. Change in rates of cerebral atrophy over time in early-onset Alzheimer's disease: longitudinal MRI study. The Lancet. 2003;362(9390):1121-2.

48. Möller C, et al. Different patterns of gray matter atrophy in early-and late-onset Alzheimer's disease. Neurobiol Aging. 2013;34(8):2014-22.

49. Lehmann M, et al. Loss of functional connectivity is greater outside the default mode network in nonfamilial early-onset Alzheimer's disease variants. Neurobiol Aging. 2015;36(10):2678-86.

50. De Waal H, et al. EEG abnormalities in early and late onset Alzheimer's disease: understanding heterogeneity. J Neurol Neurosurg Psychiatry. 2011;82(1):67-71.

51. De Waal, H., et al., Young Alzheimer patients show distinct regional changes of oscillatory brain dynamics. Neurobiology of aging, 2012. 33(5): p. 1008. e25-1008. e31.

52. Iaccarino L, et al. Spatial relationships between molecular pathology and neurodegeneration in the Alzheimer's disease continuum. Cereb Cortex. 2021;31(1):1-14.

53. Schöll M, et al. Distinct 18F-AV-1451 tau PET retention patterns in early-and late-onset Alzheimer's disease. Brain. 2017;140(9):2286-94.

54. Cavedo E, et al. Medial temporal atrophy in early and late-onset Alzheimer's disease. Neurobiol Aging. 2014;35(9):2004-12.

55. Ossenkoppele, R., et al., 2021 Accuracy of Tau positron emission tomography as a prognostic marker in preclinical and prodromal Alzheimer disease: a head-to-head comparison against amyloid positron emission tomography and magnetic resonance imaging. JAMA neurology

56. Gerritsen AA, et al. Prevalence of comorbidity in patients with young-onset Alzheimer disease compared with late-onset: a comparative cohort study. J Am Med Dir Assoc. 2016;17(4):318-23. 
57. Ortner M, et al. Small vessel disease, but neither amyloid load nor metabolic deficit, is dependent on age at onset in Alzheimer's disease. Biol Psychiat. 2015;77(8):704-10.

58. Igelström KM, Graziano MS. The inferior parietal lobule and temporoparietal junction: a network perspective. Neuropsychologia. 2017;105:70-83.

59. Engels M, et al. Alzheimer's disease: the state of the art in resting-state magnetoencephalography. Clin Neurophysiol. 2017;128(8):1426-37.

60. Brier MR, et al. Loss of intranetwork and internetwork resting state functional connections with Alzheimer's disease progression. J Neurosci. 2012;32(26):8890-9.

61. Seeley WW, et al. Neurodegenerative diseases target large-scale human brain networks. Neuron. 2009;62(1):42-52.

62. Rabinovici GD, et al. Increased metabolic vulnerability in earlyonset Alzheimer's disease is not related to amyloid burden. Brain. 2010;133(2):512-28.

63. Chen JJ, Rosas HD, Salat DH. Age-associated reductions in cerebral blood flow are independent from regional atrophy. Neuroimage. 2011;55(2):468-78.

64. Cho H, et al. Excessive tau accumulation in the parieto-occipital cortex characterizes early-onset Alzheimer's disease. Neurobiol Aging. 2017;53:103-11.
65. Vogel, J.W., et al., Four distinct trajectories of tau deposition identified in Alzheimer's disease. Nature Medicine, 2021: p. 1-11.

66. Smith R, et al. Posterior accumulation of tau and concordant hypometabolism in an early-onset Alzheimer's disease patient with presenilin-1 mutation. J Alzheimers Dis. 2016;51(2):339-43.

67. Gordon BA, et al. Tau PET in autosomal dominant Alzheimer's disease: relationship with cognition, dementia and other biomarkers. Brain. 2019;142(4):1063-76.

68. van der Flier WM, et al. Early-onset versus late-onset Alzheimer's disease: the case of the missing APOE $\varepsilon 4$ allele. The Lancet Neurology. 2011;10(3):280-8.

69. Ten Kate M, et al. Impact of APOE- $\varepsilon 4$ and family history of dementia on gray matter atrophy in cognitively healthy middleaged adults. Neurobiol Aging. 2016;38:14-20.

70. Mattsson N, et al. Greater tau load and reduced cortical thickness in APOE \&4-negative Alzheimer's disease: a cohort study. Alzheimer's research \& therapy. 2018;10(1):1-12.

Publisher's note Springer Nature remains neutral with regard to jurisdictional claims in published maps and institutional affiliations. 